\title{
Scintigraphic Evaluation of a Suspected Cavernous Hemangioma*
}

Prashant Jolepalem, MD, Catalin Grigore, CNMT, and Jane C. Palka, DO

Department of Radiology and Molecular Imaging, Oakland University William Beaumont Health Systems, Royal Oak, Michigan

$\mathbf{T}$

he patient was a 31-y-old woman with invasive ductal carcinoma of the right breast without evidence of metastases to the axillary lymph nodes. She had undergone bilateral mastectomy and was currently undergoing radiation therapy. A CT scan performed $10 \mathrm{~d}$ previously demonstrated "a $3 \mathrm{~cm}$ hypodensity within the anterior right hepatic lobe that is suspicious for a metastatic focus although benign etiology such as hemangioma cannot be excluded" (Fig. 1). A ${ }^{99 m}$ Tc-red blood cell (RBC) scan was ordered to exclude malignancy.

FIGURE 1. Transverse (liver window; left) and coronal (soft-tissue window; right) CT scans of abdomen and pelvis.
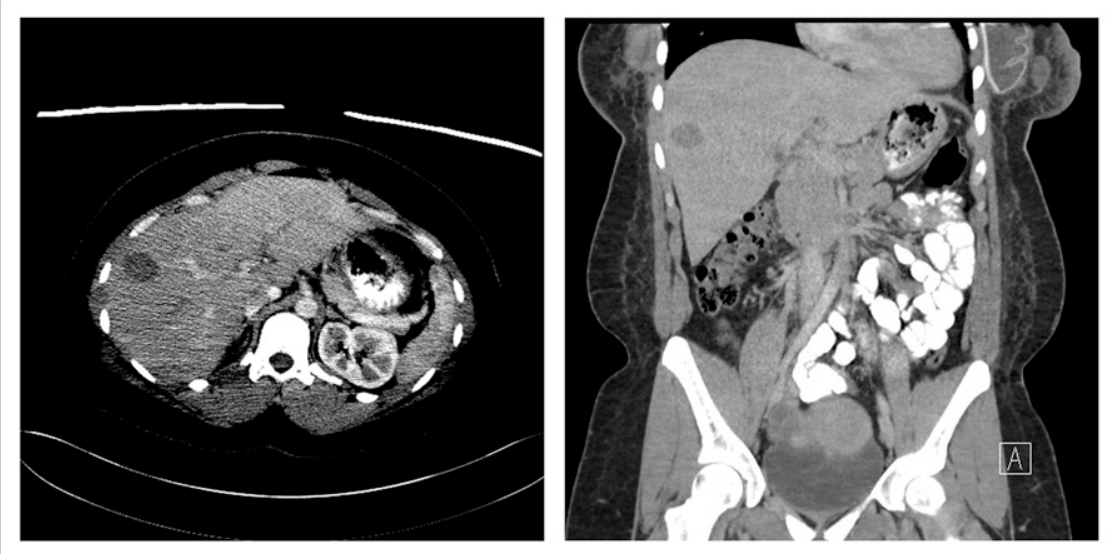

The patient was injected with $1,106.3 \mathrm{MBq}(29.9 \mathrm{mCi})$ of ${ }^{99 \mathrm{~m}} \mathrm{Tc}$ in vitro-labeled RBCs. Early and 2-h delayed planar images were taken in the anterior and posterior projections of the abdomen (heart through aortic bifurcation), with SPECT images of the early phase and SPECT/CT images of the 2-h delayed phase. Limited-field-of-view, low-dose CT was performed for anatomic localization and attenuation correction of the SPECT data.

FIGURE 2. Anterior (left) and posterior (right) early planar images.

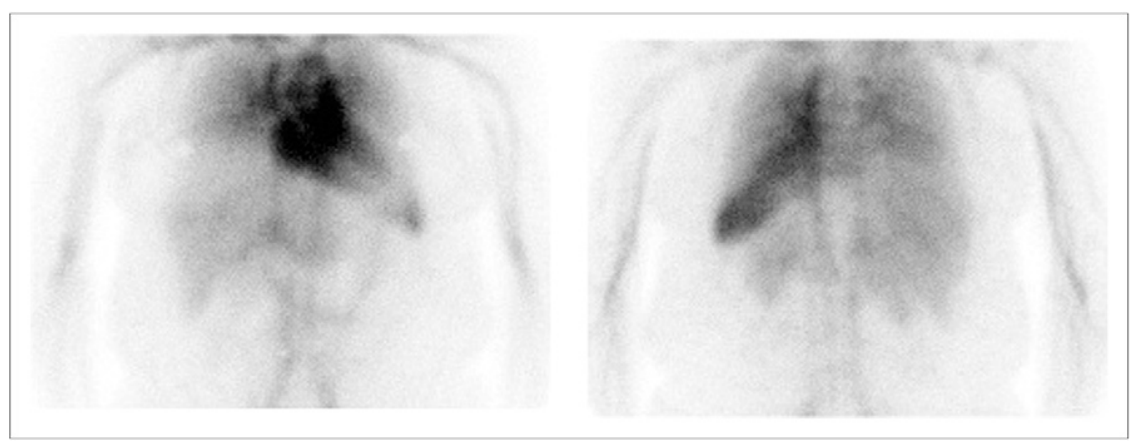

Published online Oct. 9, 2012

DOI: 10.2967/jnmt.112.114280

COPYRIGHT (c) 2012 by the Society of Nuclear Medicine and Molecular Imaging, Inc. 


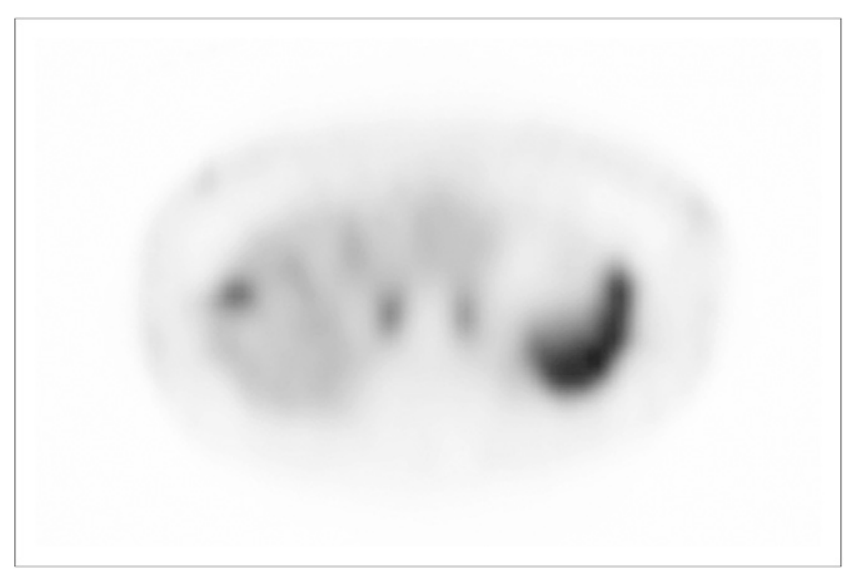

FIGURE 3. Early SPECT image.
The imaging findings were typical for a cavernous hemangioma, as the radiotracer activity during the study was consistent with the physiologic activity of RBCs within a hemangioma $(1,2)$. After the radiolabeled RBCs have had time to equilibrate, they start to enter the hemangioma and begin accumulating. The uptake may be equal to or slightly higher than in the surrounding normal liver but is still less than in the spleen, as seen on the early-phase planar images (Fig. 2) and the SPECT image (Fig. 3). On the delayedphase images, the activity within the hemangioma exceeded that within the surrounding liver and was equal to the activity within the spleen (Fig. 4). SPECT/CT proved that the focal area of uptake corresponded to the low-density lesion found on the diagnostic CT scan (Fig. 5).

SPECT alone was sufficient on the early images to localize the lesion; it was not necessary to deliver the additional radiation dose from $\mathrm{CT}$ (in accordance with the goal of

keeping the dose as low as reasonably achievable). SPECT/CT should routinely be performed on the delayed images, as it greatly increases imaging sensitivity (3). Although the SNMMI guidelines recommend performing initial flow images, this is not part of the protocol at our institution, as they are frequently of low yield (4). Typically, there would be a photondeficient area in the liver parenchyma on the initial flow images while the tracer is within the cardiac blood pool, until the hemangioma begins accumulating the labeled RBCs (1). However, if the hemangioma is in a difficult place to see on 2dimensional planar images, the absence of this finding can lead the reader astray (3).

${ }^{99} \mathrm{~m}$ Tc-RBC scintigraphy is highly specific for cavernous hemangioma (5); therefore, we consider here some differential diagnoses based on only the CT scan results. One possibility is focal nodular hyperplasia, another benign tumor that would be equivocal on ${ }^{99} \mathrm{~m}$ Tc-RBC scintigraphy and better imaged by ${ }^{99 \mathrm{~m}} \mathrm{Tc}$-sulfur colloid scanning, on which it typically shows increased uptake. Focal nodular hyperplasia can incidentally be seen on hepatobiliary imaging as well $(1,2)$.

Another possibility is hepatic adenoma. Although considered a benign tumor, hepatic adenoma is associated with lifethreatening hemorrhages. Both hemangiomas and hepatic adenomas appear as photopenic defects on ${ }^{99 m}$ Tc-sulfur colloid scans, but adenomas will be photopenic on ${ }^{99 \mathrm{~m} T c-R B C}$ scans as well $(1,2,5)$. Suspected hepatic adenomas should be imaged by MRI, where in general, they will appear hyperintense relative to background liver on T1- and T2-weighted images (3).

A third possibility is metastatic or primary malignancy. Depending on the pathology, hepatic malignancies may or may not be imaged successfully by SPECT or PET techniques. On ${ }^{99 m}$ Tc-RBC scintigraphy, malignant lesions would have normal or decreased uptake, with the exception being the extremely rare angiosarcomas, which are the only notable cause for false-positive cases $(1,5)$. MRI remains the modality of choice for characterizing suggestive hypodense lesions seen on staging CT scans (3).

\section{QUESTION 1}

For a potential hemangioma larger than $1.5 \mathrm{~cm}$, what is the modality of choice?
A. Ultrasound.
B. MRI.
C. ${ }^{99 m}$ Tc-RBC SPECT.
D. Dynamic contrast-enhanced CT.

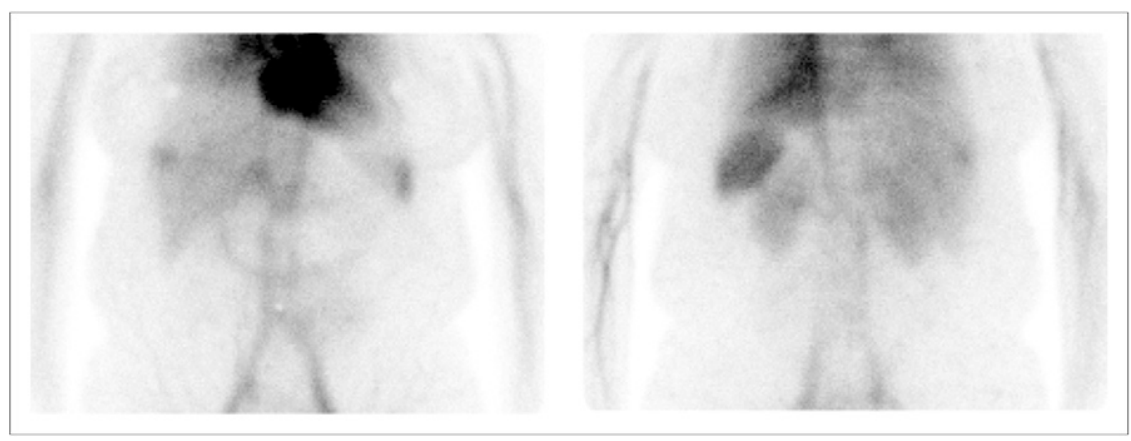

FIGURE 4. Anterior (left) and posterior (right) 2-h delayed planar images. 

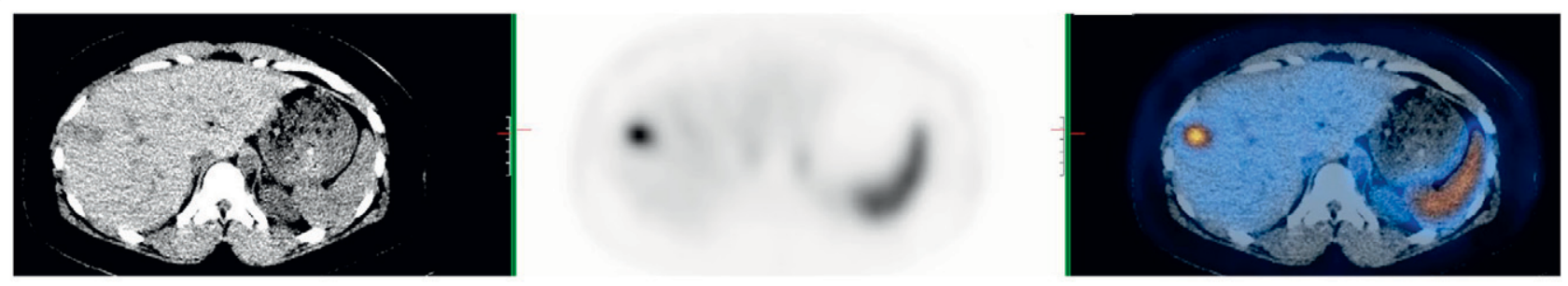

FIGURE 5. Two-hour delayed CT, SPECT, and SPECT/CT images (from left to right).

\section{QUESTION 2}

What is the typical appearance of a hemangioma on a liver-spleen sulfur colloid scan?

A. Intense circumscribed lesion.

B. Photopenic circumscribed defect.

C. Normal hepatic uptake.

D. Photopenic on early images, becoming intense on delayed.

\section{CASE DISCUSSION}

Cavernous hemangioma is the most common primary hepatic tumor. These tumors are frequently asymptomatic and incidentally discovered on imaging, surgery, or autopsy. They arise from ectasias in the endothelial lining of hepatic blood vessels and consist of multiple large, vascular channels lined by a single layer of endothelial cells and supported by collagenous walls. They are often associated with high estrogen states including puberty, pregnancy, oral contraceptive use, and steroid use. Though they are benign, hemangiomas need to be distinguished from other tumors. Rare complications include abdominal discomfort, fullness, and, rarer still, spontaneous rupture $(1,6)$.

${ }^{99} \mathrm{~m}$ Tc-RBC scintigraphy combined with multihead SPECT cameras has a nearly $100 \%$ positive predictive value. Although the sensitivity was previously dependent on size, the advent of SPECT/CT has allowed detection of hemangiomas as small as $0.5 \mathrm{~cm}$ with sensitivity of $88 \%$. The high diagnostic accuracy, low cost, and relatively low radiation dose make ${ }^{99 m}$ Tc-RBC scintigraphy a useful, although underutilized, diagnostic study for the work-up of suspected benign hepatic lesions $(3,5,6)$.

Our patient had a diagnosis of breast cancer without evidence of metastasis to the axillary lymph nodes. Therefore, the possibility of liver metastasis was considered less likely. ${ }^{99 \mathrm{~m}} \mathrm{Tc}$-scintigraphy was a low-cost, high-yield study to confirm the diagnosis of the hepatic lesion and spare the patient an unnecessary attempt at biopsy or surgical intervention.

\section{REFERENCES}

1. Ziessman HA, O’Malley JP, Thrall JH. Nuclear Medicine (The Requisites). 3rd ed. Philadelphia, PA: Mosby; 2006.

2. Mettler FA, Guiberteau MJ. Essentials of Nuclear Medicine Imaging. 5th ed. Philadelphia, PA: Elsevier Inc.; 2006.

3. Vilgrain V, Boulos L, Vullierme MP, et al. Imaging of atypical hemangiomas of the liver with pathologic correlation. Radiographics. $2000 ; 20: 379-397$.

4. Gordhan A, Soliman J, Malpani A, Peg E III. Symptomatic calvarial cavernous hemangioma. J Radiol Case Rep. 2009;3:25-29.

5. Mustafa Y, Erkan D, Oguz D. Evaluation of hepatic hemangioma with Tc-99m RBC scintigraphy: a review of 74 cases with liver masses. Turk J Gastroenterol. 2000;11:61-68.

6. Zheng JG, Yao ZM, Shu CY, et al. Role of SPECT/CT in diagnosis of hepatic cavernous hemangioma. World J Gastroenterol. 2005;11:5336-5341. 